\title{
Relato de experiência em programa multidisciplinar de atividade física e saúde
}

\author{
Report of experience in a multidisciplinary program of physical activity and health
}

Reporte de experiencia en programa multidisciplinario de actividad física y salud

\author{
Daniel Zonzini VOLTAN
}

Professor Especialista do Curso de Graduação em Educação Física do Centro Universitário de Jaguariúna (UniFAJ) 13820-000 Jaguariuna - SP, Brasil Mestrando em Psicologia pela Pontifícia Universidade Católica de Campinas (PUCCAMP)

\section{Resumo}

Introdução: As atuações multidisciplinares na área da saúde têm sido cada vez mais constantes e eficientes. Propor modelos de aplicação deste tipo de intervenção contribui sobremaneira para o desenvolvimento de ações em todos os setores de atendimento em saúde. Objetivo: Descrever as atividades de um profissional de Educação Física em uma equipe multidisciplinar no atendimento de saúde "Programa Viver Bem - Interclínicas no campus III do Centro Universitário de Jaguariúna”. Método: Relato de experiência a partir do método self-study. Discussão: A trajetória deste profissional foi composta de diversos projetos sociais e educacionais, atuando a partir das funções enquanto professor/orientador/gestor do programa no oferecimento de atividades físicas. Conclusão: O trabalho multidisciplinar e a convivência com outros profissionais da área da saúde como Enfermagem, Fisioterapia, Nutrição e Psicologia foram de fundamental importância para o sucesso do programa e pela excelência nos seus atendimentos.

Descritores: Educação Física e Treinamento; Planos e Programas de Saúde; Equipe de Assistência ao Paciente.

\section{Abstract}

Introduction: The multidisciplinary actions in the health area have been increasingly constant and efficient. Proposing models of application of this type of intervention contributes greatly to the development of actions in all sectors of health care. Objective: To describe the activities of a Physical Education professional in a multidisciplinary team in the health care "Programa Viver Bem - Interclínicas no campus III do Centro Universitário de Jaguariúna". Method: Report of experience from the self-study method. Discussion: The trajectory of this professional was composed of several social and educational projects, acting from the functions as teacher / supervisor / manager of the program in the offering of physical activities. Conclusion: The multidisciplinary work and the coexistence with other health professionals such as Nursing, Physical Therapy, Nutrition and Psychology were of fundamental importance for the success of the program and for excellence in its services.

Descriptors: Physical Education and Training; Health Programs and Plans; Patient Care Team.

\section{Resumen}

Introducción: Las actuaciones multidisciplinares en el área de la salud han sido cada vez más constantes y eficientes. Proponer modelos de aplicación de este tipo de intervención contribuye sobremanera para el desarrollo de acciones en todos los sectores de atención en salud. Objetivo: Describir las actividades de un profesional de Educación Física en un equipo multidisciplinario en la atención de salud del "Programa Viver Bem - Interclínicas no campus III do Centro Universitário de Jaguariúna". Método: Relato de experiencia a partir del método self-study. Discusión: La trayectoria de este profesional fue compuesta de diversos proyectos sociales y educativos, actuando a partir de las funciones como profesor / orientador / gestor del programa en el ofrecimiento de actividades físicas. Conclusión: El trabajo multidisciplinario y la convivencia con otros profesionales del área de la salud como Enfermería, Fisioterapia, Nutrición y Psicología fueron de fundamental importancia para el éxito del programa y por la excelencia en sus atenciones.

Descriptores: Educación y Entrenamiento Físico; Planes y Programas de Salud; Grupo de Atención al Paciente.

\section{INTRODUÇÃO}

O trabalho em equipe deve ser considerado uma modalidade coletiva de relação mutual entre as ações técnicas específicas e a interação de seus autores. Baseia-se na construção de consensos que estruturam um projeto assistencial em comum ${ }^{1}$.

Consonante com esta perspectiva, a multidisciplinaridade apresenta-se numa prática utilizada especialmente na área da Saúde ${ }^{2}$, norteada pela aproximação de diferentes disciplinas para a solução de problemas específicos, pela diversidade de metodologias de cada disciplina e dos campos de atuação, que mesmo em cooperação, possuem delimitações marcadas e muitas vezes não se comunicam $^{3}$. Tem ainda por objetivo atender à todos os aspectos bio-psico-físico-sócio-culturais do indivíduo ${ }^{4,5}$.

Dessa forma, propor ações neste formato na respectiva área pode contribuir para o desenvolvimento saudável de uma população. Portanto, relatar estas ações contribuem na formação de um arcabouço teórico interligado diretamente com a prática mostrando suas possibilidades, viabilidades e experiências exitosas a serem pensadas e implantadas por outros.

Exemplo destas ações enquanto extensão universitária, são trabalhos dentro do contexto de atendimento advindos do Sistema Único de Saúde com atenção secundária à saúde ${ }^{6}$, ou especificamente nas Unidades Básicas de Saúde ${ }^{7}$. Existem também aqueles voltados para uma determinada população ${ }^{8}$ ou ainda em ações em ambientes como as Unidades de Tratamento Intensivo 9.

Assim, o objetivo deste trabalho é relatar como ocorreram as dinâmicas de atendimento do Programa Viver Bem - Interclínicas FAJ para a população de Jaguariúna-SP.

\section{MATERIAL E MÉTODO}

Esta pesquisa qualitativa possui caráter exploratório-descritivo com o intuito de relatar as experiências mais marcantes da vivência do autor dentro do referido programa.

Para isso, foi pensado a utilização de abordagem metodológica rigorosa e ao mesmo tempo que permita articular as impressões pessoais. Assim, self-study foi o método escolhido para este trabalho, entendido como uma forma de pesquisa do profissional concentrado especificamente na prática autodeclarada ${ }^{10,11}$. Além disso, os pesquisadores deste método compartilham um conjunto comum de 
características e disposições: este profissional é membro de uma comunidade profissional que compartilha, pesquisa e desenvolve sua própria prática; cada pesquisador assume uma postura orientada pela investigação para pesquisar como ele próprio pensa, conhece e age nos contextos em que estão inseridos; ao transformar o foco da investigação no self, o pesquisador do self-study expressa implicitamente o desejo de melhorar a si próprio a partir das suas reflexões e novos autoentendimentos ${ }^{11}$. Prova disso é que "o propósito do self-study [é] ir além das particularidades da prática, tornando públicos os entendimentos desenvolvidos (através de apresentações em conferência, relatórios de pesquisa, manuscritos do jornal) para torná-los informativos para os outros e disponiveis para o debate crítico ${ }^{, 12}$.

Para isso, este trabalho foi dividido em "O Programa" que descreve o espaço institucional e as ações desenvolvidas a partir do seu projeto práticopedagógico, e "Da minha atuação", que analisa as ações do autor junto ao programa de acordo com os movimentos institucionais impostos e reflexões a partir do self-study, uma vez que suas decisões influenciaram na dinâmica da relação coordenador/professor do programa/beneficiários.

\section{RESULTADOS E DISCUSSÃO}

\section{$>$ O Programa}

Criado em 2009 pelo curso de Educação Física da instituição de ensino superior (IES) Faculdade de Jaguariúna (FAJ), pretendia proporcionar um serviço gratuito e especializado de atividade física sob o prisma da profilaxia. Pelo fato da Interclínicas (Campus III) estar localizada no bairro Roseira de Baixo, na cidade de Jaguariúna-SP, o então chamado Projeto Bem Viver Roseira atendia inicialmente os moradores idosos ( $\geq 60$ anos) desta região da cidade.

Em 2010, o Programa aumentou sua capacidade de atendimento passando a receber idosos de toda a cidade. No ano seguinte, os critérios para atendimentos valeriam para qualquer pessoa com idade mínima de 20 anos e intitulou-se então Programa Viver Bem - Interclínicas FAJ (PVB) devido às outras ações implantadas.

O objetivo do programa foi proporcionar prevenção e promoção da saúde através de exercícios físicos orientados, ações sociais, culturais e psicoeducativas com foco na qualidade de vida da população adulta e idosa de Jaguariúna-SP.

Além disso, foi um campo de estágio para os alunos dos cursos da área de Saúde. No caso dos futuros professores de Educação Física, este campo de experiências possibilitou a construção dos saberes pedagógicos a partir das necessidades pedagógicas postas pelo real ${ }^{13}$.

O PVB se desenvolveu multidisciplinarmente na Educação Física, Psicologia, Nutrição, Fisioterapia e Enfermagem. Cada uma dessas áreas atuou em momentos específicos dentro das ações desenvolvidas graças à necessidade de integrar diversos conhecimentos científicos e práticas diferentes para atender as demandas da população.

Dentro do PVB várias ações foram desenvolvidas com objetivo de atender as demandas estabelecidas pelo Plano de Desenvolvimento Institucional (PDI) ${ }^{14}$ desta IES. Tais quais, ensino-pesquisa-extensão. Estas ações ocorreram da seguinte maneira:

- Academia Interclínicas

Contou com atendimento de hidroginástica com mais de 20 usuários na piscina. Também possuiu aulas de alongamento com o mesmo número de usuários. E, por fim, ofereceu exercícios com peso em sala de musculação. Cada atendimento foi oferecido 2 vezes por semana, e os usuários podiam frequentar até 2 atendimentos.

\section{- Grupo de Obesidade Grau 3 (GOBE III)}

Criado com o objetivo de atender duas vezes semanais aquelas pessoas que apresentavam grau de obesidade mórbido (IMC $\geq 40)$ e precisavam de treinamento, dieta, motivação e cuidados específicos. Neste grupo, o trabalho multidisciplinar entre Educação Física na prescrição de exercícios eficientes, Nutrição na prescrição de dieta e Psicologia no suporte e motivação estavam alinhados para obter melhores respostas quanto ao estado de saúde dos indivíduos. Em vários momentos, as intervenções aconteciam simultaneamente durante a seção.

\section{- Viver Bem Recreando}

Estagiários do curso de graduação em Educação Física realizaram atividades lúdicas e recreativas juntamente com o Projeto de Atendimento à Criança do curso de Enfermagem. Os estagiários de ambos os cursos atuaram com o propósito de oferecer atividades recreativas educacionais para as crianças dos bairros Roseira de Baixo e Roseira de Cima visando o bem-estar, saúde e educação através de jogos e brincadeiras.

\section{- Projeto Movimento Inclusivo (MOVI)}

A partir da necessidade de exercício físico específico e continuidade do processo de reabilitação, pacientes atendidos pelo curso de Fisioterapia foram encaminhados para este projeto. Estes pacientes recebiam treinos adaptados à suas capacidades e condições físicas.

O resultado disso foi a recuperação da autoestima, capacidades físicas e habilidades motoras que antes não se imaginavam possíveis e consequentemente, a adesão à prática de exercícios físicos.

Foram atendidos neste projeto indivíduos com paraplegia, amputação e perda de movimento de membros superiores por trauma. Em encontros informais posteriores, estes usuários continuam 
ativos, trabalhando e treinando, "graças à este projeto" segundo eles.

- Grupo de Estudos de Atividade Física e Saúde (GREAFIS)

Por se tratar de um programa multidisciplinar os estagiários eram motivados a participar deste Grupo de Estudos. A partir de um cronograma de atividades, dinâmicas, palestras e estudos voltados para este tema, os alunos dos estágios de Fisioterapia, Educação Física, Nutrição, Enfermagem e Psicologia recebiam e compartilhavam informações e atualizações a partir da perspectiva do trabalho multidisciplinar.

Fruto deste grupo foi a realização de uma avaliação do peso, estatura, IMC, hábitos alimentares e estilo de vida nos alunos da rede municipal de ensino, em parceria com a Prefeitura Municipal de Jaguariúna através da sua Secretaria de Educação. A partir destas coletas foram realizados 2 trabalhos de conclusão de curso ${ }^{15,16}$ na Educação Física e a apresentação de um relatório final para a Secretaria de Educação de Jaguariúna ${ }^{17}$.

\section{$>$ Da minha atuação}

Este Programa me propiciou grande desenvolvimento profissional e humano. Durante minha gestão acumulei funções que foram fundamentais para subsidiar as disciplinas que leciono no ensino superior. E, comprovar através desta experiência a extrema necessidade e efetividade da tríade ensino-pesquisa-extensão ${ }^{18}$ enquanto alicerce para formação de profissionais capacitados e autônomos. Assim, foi possível dividir meu relato sob três aspectos da minha função:

\section{○ Eu-orientador}

Este processo ocorreu de forma tranquila, pois tinha todo o respaldo da IES. Facilitou muito a adequação das documentações obrigatórias para os graduandos, uma vez que o estágio acontecia no próprio campus.

Os estagiários puderem trabalhar e aprender com realidades de saúde diferentes tornando-se professores de Educação Física mais preparados para o mercado de trabalho e seus saberes pedagógicos maiores.

\section{○ Eu-professor}

Para um professor o aprendizado nunca acaba. Existe uma carência, especialmente no idoso, de brincar, de ser ouvido, de poder se exercitar, de mostrar-se ativo e de interação interpessoal. Pude então, atender algumas dessas demandas e isto me trouxe um novo olhar para minha formação. Pois, todos que procuraram algum atendimento do Programa, apresentavam alguma(s) patologia(s). Este conteúdo no Programa certamente dará maior suporte às prescrições dos profissionais desta área que ali estagiaram.

\section{○ Eu-gestor}

Enquanto gestor, pude prender a administrar os trâmites institucionais e fazer grandes interações com profissionais de outros cursos da área da saúde me possibilitando ver a Saúde de forma macroambiental. Enquanto gestor é óbvio que a eficiência dos atendimentos era uma diretriz explícita da instituição.

Muitas vezes, essas decisões criavam ou desativavam algum atendimento que os usuários gostavam muito. Este processo fez com que minhas habilidades de gerenciamento de conflitos aumentassem exponencialmente.

Além disso, desenvolver ações multidisciplinares me forçou a conhecer em profundidade as intervenções destes outros profissionais, como explorar os conhecimentos de cada um para oferecer o melhor atendimento e refletir ainda mais sobre as minhas ações/atuações.

Claramente algumas decisões enquanto gestor afetava o meu relacionamento enquanto professor. A relação (usuário)aluno-professor, especialmente entre adultos, não se limitam na hierarquização da relação, mas transcende para amizades duradouras e ultrapassa os limites da fronteira do programa.

$\mathrm{O}$ aspecto da socialização sempre esteve presente entre os usuários, tanto que os grupos de atendimentos possuíam características distintas, tal qual uma identidade de grupo.

$\mathrm{O}$ relato de experiência apresentado no presente trabalho compreendeu o período entre 11 de fevereiro de 2011 e 30 de junho de 2014. Durante este período o Programa sofreu várias modificações adaptando-se às realidades dos usuários e aos alunos da IES e muitos objetivos foram alcançados. Atendeu-se com dignidade e conhecimento a população de Jaguariúna. Foram realizados 14.262 atendimentos para 287 usuários. Mais de 10 estagiários permanentes da Educação Física passaram pelo Programa. Turmas inteiras de psicologia e alunos de vários semestres de nutrição puderam participar e vivenciar ativamente nos ações que o PVB disponibilizou adquirindo conhecimento e experiência ímpar devido à sua proposta prática multidisciplinar.

CONCLUSÃO

O trabalho e a convivência com outros profissionais da área da saúde como Enfermagem, Fisioterapia, Nutrição e Psicologia foram de fundamental importância para o sucesso do PVB e pela excelência nos seus atendimentos mostrando a eficiência da multidisciplinaridade.

Os usuários, principais beneficiados, puderam usufruir de um equipado centro de treinamento para as capacidades físicas num ambiente alegre, acolhedor e respeitoso.

\section{AGRADECIMENTOS}

Às ações sempre dispostas e otimistas da 
psicóloga Prof. Esp. Marcela Gonçalves Soares di Tílio, a atuação diária da nutricionista Prof. Esp. Natália Conessa, o suporte e disposição da educadora física Prof. Esp. Jaqueline Dell Vecchio Barbosa e às coordenadoras dos cursos de Nutrição, Psicologia e Enfermagem que sempre se mostraram presentes nos momentos de dúvidas e motivaram cada fase do Programa, muito obrigado. E, agradeço especialmente a idealizadora deste programa, a então coordenadora do curso de Educação Física, Prof. Beatriz Leme Passos de Carvalho.

\section{REFERÊNCIAS}

1. Peduzzi M. Equipe multiprofissional de saúde: a interface entre trabalho e interação. Interface (Botucatu). 2000;4(6):151-51.

2. Garcia V. Visão Interdisciplinar e Multidisciplinar dos Profissionais na Área da Saúde. Brasil: World Gate; 2007.

3. Domingues I. Em busca do método. In: Domingues I (ed) Conhecimento e transdisciplinaridade II: aspectos metodológicos. Belo Horizonte: Editora UFMG; 2005.

4. Mauss M. As técnicas corporais. In: Mauss M (ed). Sociologia e antropologia - vol 2. São Paulo: Edusp; 1974.

5. Bronfenbrenner U. Making human beings human: bioecological perspectives on human development. Thousand Oaks: Sage Publications; 2005.

6. Novo Júnior JM, Silva LP, Chicourel EL, Lima JRP, Matta MO, Peralva MAC. As características das ações multidisciplinares do programa de caminhada orientada da UFJF na atenção integral da saúde. Interagir. 2006;10:61-6.

7. Silva MP, Santos JW, Souza AN, Tempesta SD, Avelino RA. Programa multidisciplinar para promoção da saúde envolvendo atividade física supervisionada: ações do PAFIPNES na atenção à saúde de mulheres em uma Unidade Básica de Saúde de São José do Rio Pardo-SP. Rev bras ativ fis saúde. 2011;16(4):362-66.

8. Alves Rezende MCR, Capalbo BC, Soubhia AMP, Poi WR, Fajardo RS, Bresciani KDS, et al. UNATI - promoção de qualidade de vida na terceira idade. Arch Health Invest. 2012;1(1Suppl. 1):67.

9. Squive RC, Borges SM, Silva A de M, Silva JM da, Oliveira MP de, Passos BB et al. Atuação do cirurgião-dentista em equipe multidisciplinar no cuidado ao paciente crítico. Arch Health Invest. 2017;6(Special Issue 3):101.

10. Hamilton ML, Pinnegar S. Conclusion: The value and promise of self-study. In: Hamilton ML (ed). Reconceptualizing teaching practice: Self-study in teacher education. London: Falmer Press; 1998. p. 235-46.
11. Ovens A, Fletcher T. Doing self-study: The art of turning inquiry on yourself. In: Ovens A, Fletcher $\mathrm{T}$, editors. Self-study in physical education teacher education: The interplay of practice and scholarship. Dordrecht: Springer; 2014. p. 3-14.

12.Vanassche E, Kelchtermans G. The state of the art in Self-Study of Teacher Education Practices: a systematic literature review. J Curriculum Stud. 2015;47(4):508-28.

13.Pimenta $S G$ (ed). Saberes pedagógicos e atividade docente. 3.ed. São Paulo: Cortez; 2002.

14.Faculdade de Jaguariúna. Plano de Desenvolvimento Institucional: PDI para o período 2012 - 2016. Jaguariúna; 2012.

15. Nascimento CF, Lança G, Fontes JA, Campos LH. Obesidade Infantil Escolar. Faculdade de Jaguariúna; 2013.

16. Rolfsen C, Bárbaro EL, Boveri RA. Análise do IMC em alunos das escolas municipais de Jaguariúna-SP. Faculdade de Jaguariúna; 2013.

17. Voltan DZ, Carvalho BLP. Relatório Saúde do Escolar Jaguariúna 2013. Jaguariúna; 2013.

18. Gonçalves NG. Indissociabilidade entre Ensino, Pesquisa e Extensão: um princípio necessário. Perspectiva. 2015;33(3):1229-56.

\section{CONFLITO DE INTERESSES}

O autor declara não haver conflitos de interesse.

\section{AUTOR PARA CORRESPONDENCIA}

\section{Daniel Zonzini Voltan}

danielzvoltan@gmail.com

Submetido em 16/06/2018

Aceito em 04/10/2018 